\title{
High Dynamic Range Variable Optical Attenuation (Project Report CIAN2-1-Y5)
}

\section{SHAYAN MOOKHERJEA}

Department of Electrical and Computer Engineering, University of California, San Diego, MC 0407 La Jolla CA 92093-0407 USA

Email:smookher@ucsd.edu

\section{Introduction and Goals}

The research activities reported here were performed under Thrust 2 "Subsystem Integration and Silicon Nanophotonics" of the NSF-funded Center for Integrated Access Networks. The goal of this project was to design silicon photonic components for optical WDM (wavelength division multiplexed) add/drop functionality in access and data networks. One of the key functionalities that was required by the campus networking testbed was to control the optical power levels in the WDM network of each channel without requiring separate components. This was most easily achieved by integrating the Variable Optical Attenuator (VOA) functionality together with the add-drop functionality on the same photonic chips (see Fig. 1). Each of the six network nodes was responsible for controlling the power levels of the four channels it adds to the WDM network, and also control the power of the "express" channel which contained all the other WDM channels.

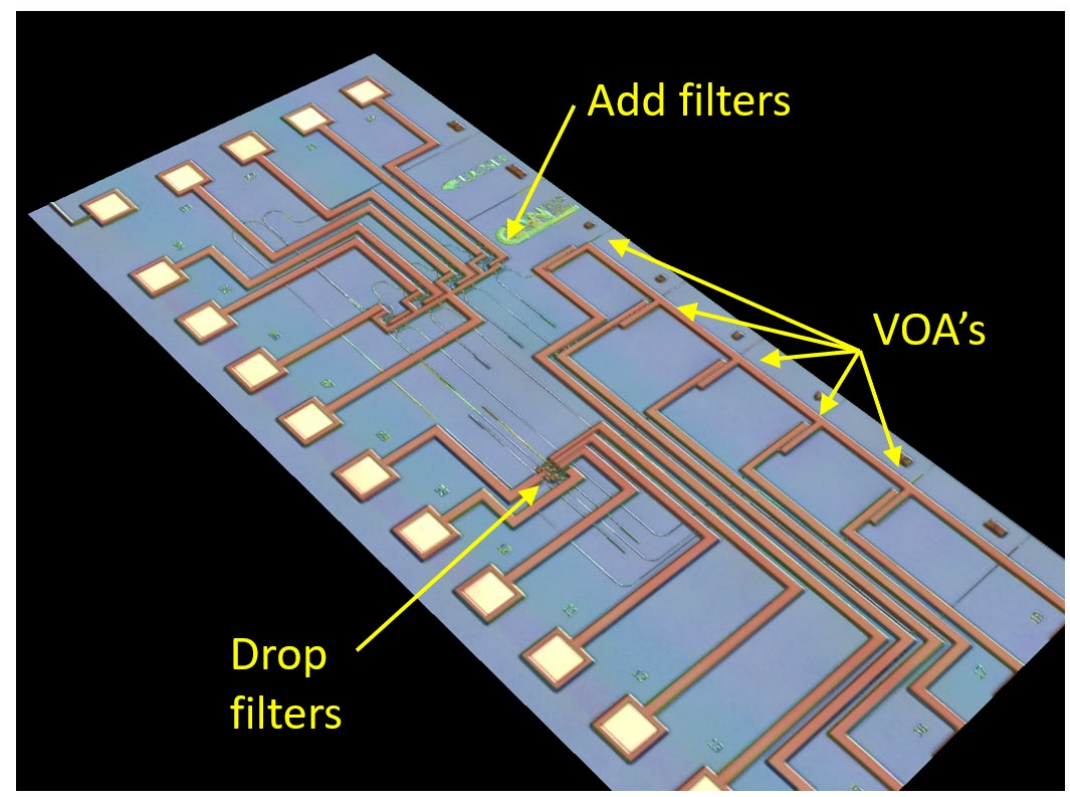

Figure 1 Laser scanning microscope image of silicon photonic chip which provides add/drop functionality and variable optical attenuation (VOA) functionality for up to four WDM channels. The chip was fabricated by Sandia National Labs as part of a multi-project wafer fabrication project with a group of university collaborators. 


\section{Activities}

We published a report on the performance of the first-generation add/drop microchip [1] as tested on a 24-channel wavelength division multiplexed (WDM) optical network testbed "MORDIA" at UC San Diego [2]. In the second-generation microchip, the add-drop functionality was provided using higher-order coupled-microring filters, rather than simple microring resonators. The same chip design could be used by any of the nodes, to address a group of four wavelengths throughout the $\mathrm{C}$ band. The filters were tunable over the entire $\mathrm{C}$-band using thermal tuning of the microrings. Furthermore, electronic control over the optical power level of each of the added channels and the through "express" channel that passes through each chip was achieved by using waveguide-integrated variable optical attenuators (VOAs).

These VOA's were formed using $\mathrm{p}$-n junctions fabricated across ridge silicon waveguides, which are lightly p-doped. The structure consists of a p-n doped diode structure fabricated across a partially-etched silicon waveguide, as shown in Figure 2. Under forward bias, a current in the range of $0-100 \mathrm{~mA}$ is injected and the presence of a large concentration of free carriers around the waveguide mode causes optical attenuation ("off" state). Under reverse bias, the junction is depleted of carriers, the optical attenuation is reduced ("on" state). A rib-toridge transition is used in the silicon layer to facilitate the transition from fullyetched waveguides to the slab waveguide structure that is needed for the VOA. This is shown in Figure 3 (left panel).

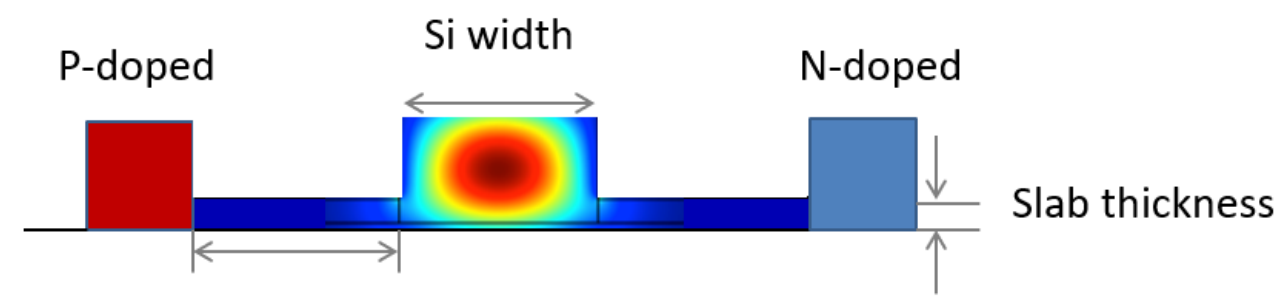

Figure 2 Schematic of the waveguide cross-section incorporating a p-n diode as used for a Variable Optical Attenuator (VOA). Current injection under forward-bias conditions causes an increase in the optical propagation loss. The structure is designed to have a low optical loss when there is no forward bias voltage applied.

The device was designed on a silicon photonics process at the Sandia National Laboratories on a silicon-on-insulator wafer with $230 \mathrm{~nm}$ active-layer silicon thickness and $3 \mu \mathrm{m}$ buried oxide (BOX). The slab tapers shown in Fig. 3 measured $150 \mathrm{~nm}$ in thickness, and the doped regions were of length $50 \mu \mathrm{m}$. The dopants had a $900 \mathrm{~nm}$ separation from the edge of the intrinsic waveguide core, which was $400 \mathrm{~nm}$ wide.

Figure 3 shows the improved performance in the variable optical attenuator (VOA). Using about the same level of current injection into the VOA as in the first-generation device, a much higher dynamic range could be achieved in the revised design. Previously, increasing the current in the VOA resulted in device 
damage, which is common when passing more than $100 \mathrm{~mA}$ through narrow structures.

This was due to an innovative design in which two stages were used to achieve the VOA functionality. First, the diode was used in forward-bias mode to attenuate the light. Current flow through a small diode causes heating, and the heat spreads along the thin silicon device layer since the underlaying oxide acts as an effective barrier. An integrated optical filter, consisting of a pair of coupled microring resonators, was tuned thermally to gradually move onto resonance with the wavelength. In its initial configuration, the filter was offresonance with the wavelength of the light transmitted through the VOA. As the VOA was heated up by the injected current, the heat caused the filter to redshift and move closer to resonance. This further attenuated the transmitted light, since some of the light was coupled into the microring resonator and extinguished. The net effect was that a higher total absorption could be achieved without damaging the diode by passing a higher current through it.

Of course, such a scheme only works at certain wavelengths, but we have previously shown that the microrings that were used on this chip have usable resonances with $>20 \mathrm{~dB}$ dynamic range throughout the $\mathrm{C}$-band. Since the VOA's are used at the input ports (as part of the "add" section), they only address one wavelength at a time. Therefore, this scheme is suitable for use in this particular application. For general usage, the simple diode structure on its own is more suitable for optical power control over a broad spectrum.
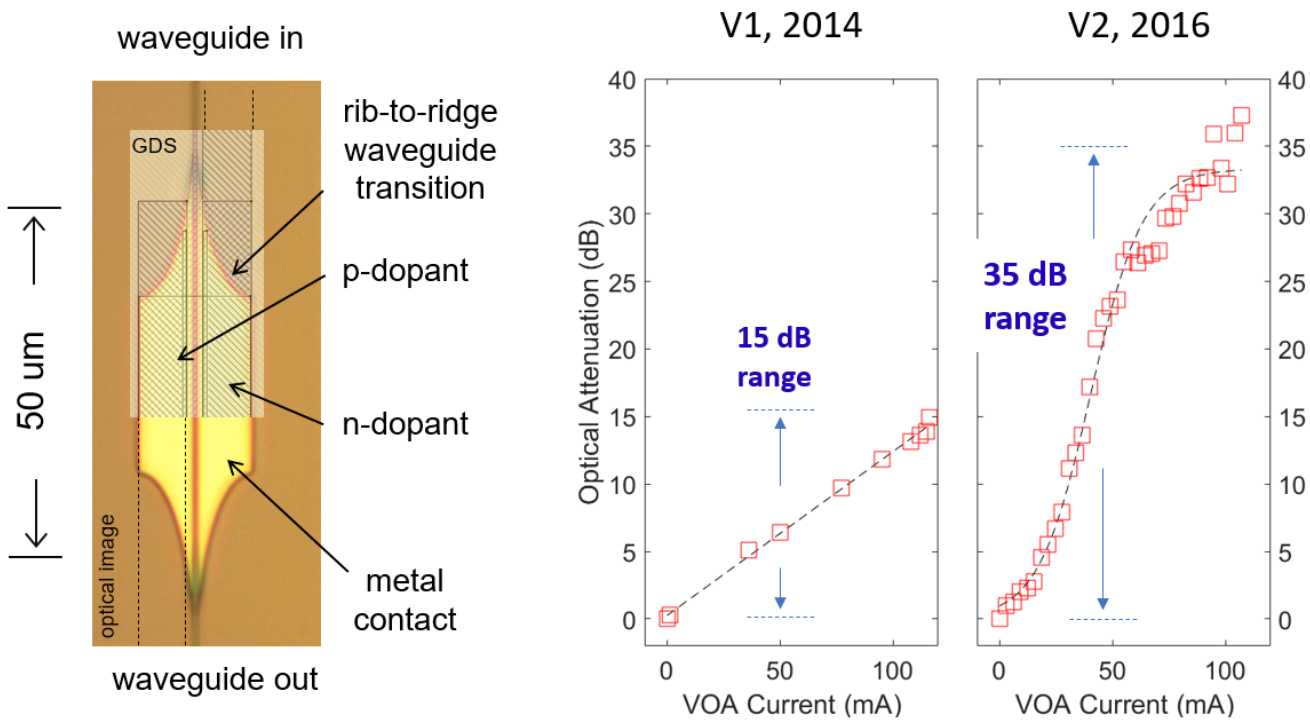

Figure 3 Improved performance in the Variable Optical Attenuator in the second-generation design, resulting in a higher optical attenuation range over the same range of current injection.

The filter that followed the diode-based VOA stage used a pair of coupled silicon microrings with directional couplers. Three directional couplers are 
required with two microrings: one between the rings, and one on either side of the filter to couple to bus waveguides on the input and drop side. To achieve the correct bandwidth and free spectral range (FSR), we had experimentally studied the dispersion of directional couplers using a microring coupled to a waveguide [3]. An accurate knowledge of dispersion is also necessary for a wide range of integrated photonics devices, such as coupled-microring filters [4].

\section{Progress and Impact}

The benefit of this architecture, when it can be used, is that it relaxes the constraints on achieving a high on-off ratio (here, about $40 \mathrm{~dB}$ ) by using only one type of structure.

A single-stage $\mathrm{p}$-i-n attenuator will have to be made longer in order to increase the usable range of optical attenuation from about 15-20 dB (as shown in our first generation devices), to $40 \mathrm{~dB}$ and this will increase the insertion loss even in the "off" state. Alternatively, a much higher voltage may be required, which can damage the diode as the current is exponentially related to voltage above threshold, according to the well-known diode equation.

On the other hand, achieving high transmission contrast by only using a microring can be problematic since the transmission characteristics depend on optical input power to a much greater degree than the $p-i-n$ attenuator. It is difficult to achieve a $40 \mathrm{~dB}$ contrast using a bus-coupled microring unless the deviation from critical coupling is very small. In a group or four of more structures, there will be significant differences in performance due to disorder. In contrast, Figure 4 shows that several different two-section VOA structures placed at different parts of an optical circuit achieved similar performance.

Coupled microrings were used to achieve a more versatile and tunable filtering response compared to single microring resonators. They can achieve a flat-top transmission passband with steeper sidewalls. These measurements were performed by tuning one resonator in a coupled-resonator system slightly away from the other. Previously, we have developed the theory and design principles of coupled microring resonators, also known as Coupled Resonator Optical Waveguides (CROWs). A tight-binding model of band-center propagation was formulated [5], and theoretical studies of nonlinear propagation were developed on this basis [6]. Studies were performed to examine the regions of high dispersion which may be relevant to signal distortion when filtering wideband signals [7] or all-optically tunable dispersion compensation devices [8]. To help in designing structures in silicon photonics, we have developed an extension of coupled-mode theory which is applicable to directional couplers in high index contrast materials, in coupled-waveguide structures [9] and coupled-resonator structures [10]. Although a second-order filter structure was used here, the 
concept could be expanded to use a higher-order filter with a modest number of resonators (for example, about five).

A collaboration with IBM's silicon photonics group (Dr. W. M. J. Green) has provided insights into industrial silicon photonics fabrication methods [11], [12]. Dr. Ivan B. Divliansky (CREOL, Florida) collaborated with us for the electron-beam lithography of long waveguide structures [13]. As part of collaborative projects supported by the NSF, CROWs of up to 235 coupled resonators were made and measured [12], [14], [15].

Impact on Human Resources: Two graduate students in the PI's group worked on this topic as part of their education and training. The Center provided extensive opportunities to interact with researchers and students from other groups and universities as well as with researchers from Sandia.

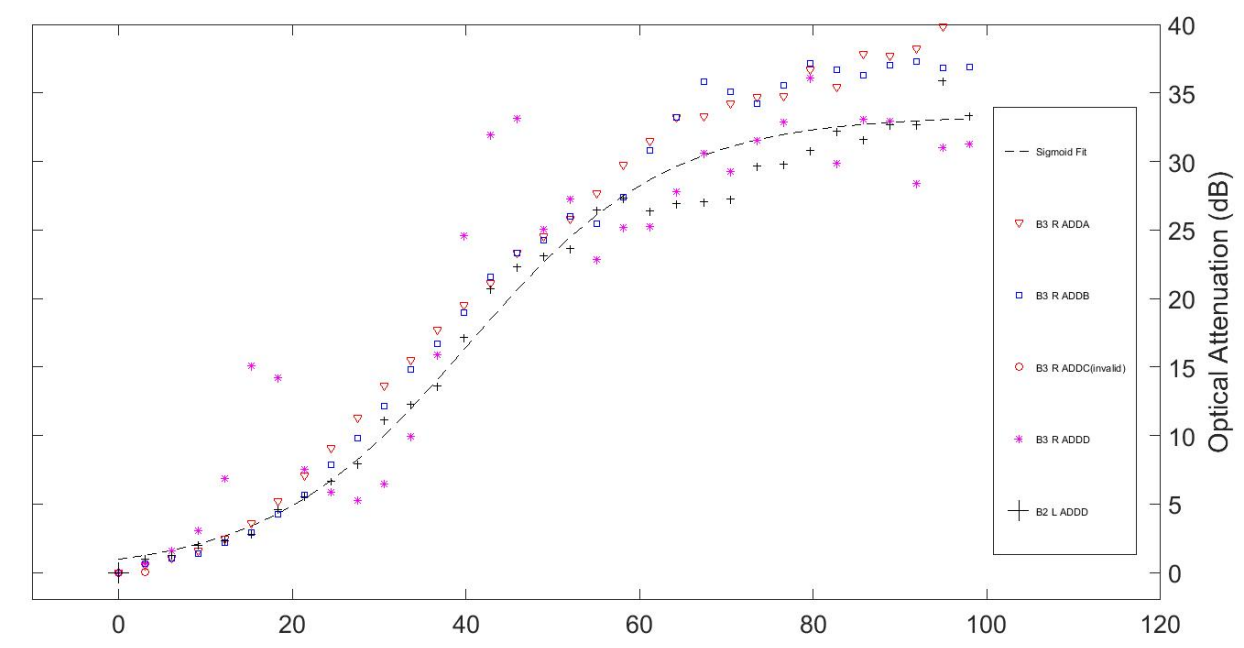

Figure 4 Studies on a batch of different two-stage VOA devices across a die, showing that the general trend was the same across different devices. The horizontal axis is electrical current $(\mathrm{mA})$, and the vertical axis is the optical attenuation $(\mathrm{dB})$.

\section{Subsequent Extensions}

CROW structures can have other applications in photonics, such as the generation of entangled photon pairs [16], and developing novel amplifiers and lasers [17]. For example, the resonator-enhanced nonlinearity benefits the generation of entangled photon-pair and heralded single-photon generation using silicon photonics [18]. Our initial measurements of spontaneous fourwave mixing (SFWM) were on correlated photon-pair generation and heralded single-photon generation. An experimental measurement of entanglement was performed later, using a two-photon interferometer constructed to verify timeenergy entanglement [18]. Structures such as VOA's can be useful in improving the amplitude balance between different optical pathways on a microchip circuit. VOA's in silicon photonics can be tuned rapidly (at sub nanosecond 
speeds) are also useful to protect sensitive detectors from unwanted levels of input optical power, such as the so-called "blinding attacks" on single-photon avalanche diodes.

These microring filters are tuned using the thermo-optic effect. Thermal tuning, rather than current injection, of microrings results in a higher achievable on-off contrast because current injection induces free carrier absorption in the microrings (enhanced by the multiple round trips made by light in the resonator).

Although the tuning speed was greatly improved from milliseconds to microseconds, there are some applications that require even faster tunability. For such applications, electronic controllability can be of interest, especially in the coupled-microring structure [19]. Carrier-driven effects can achieve subnanosecond speeds of varying the optical index and absorption. Even faster effects can be achieved using ferro-electric materials which decouple the amplitude and phase effects. For example, optically-transparent thin-film ferro-electric materials bonded to silicon waveguides can be used to achieve phase shifters with more than $100 \mathrm{GHz}$ of bandwidth, as was demonstrated later [20].

Microring resonators are sensitive to disorder. As a way to compensate for defects, rather than rely solely on thermo-optic heaters for compensatory tuning, we studied techniques for precisely tuning resonators. One method we studied is based on field-induced local oxidation of $\mathrm{Si}$ to $\mathrm{SiO}_{2}$ via a chemical reaction near an electrically-biased conducting atomic-force microscope (AFM) tip [21]. While this method achieves extremely precise control over the resonance frequency, it is a slow process since the AFM tip scans over nanometer-scale regions at the few-second time scale. In practice, a single silicon microresonator can be monitored and controlled more easily [22] than a device that consists of many coupled resonators.

Another experimental technique that was useful in this work and other projects was infrared imaging. Images acquired using a microscope objective and InGaAs camera were used to study light propagation through optical circuits [13], [23] and the images can be used to obtain information about resonators and couplers.

\section{Open-Access Reporting Initiative}

PRAISE: This open-access document is provided in support of our PRAISE (Public Report of Activities, Impact and Subsequent Extensions) initiative. What is it? An open-access document shared with the public which describes the research outcomes of publicly-funded projects. For us, these projects are typically funded by the NSF (National Science Foundation). 


\section{References}

[1] R. Aguinaldo et al., "Silicon photonic integrated components for add, drop, and VOA in a 4-channel data-center network," in 2014 IEEE

Photonics Conference, San Diego, CA, Dec. 2014, pp. 1-2. doi: 10.1109/IPCon.2014.7092976.

[2] R. Aguinaldo et al., "Wideband silicon-photonic thermo-optic switch in a wavelength-division multiplexed ring network," Opt. Express, vol. 22, no. 7, p. 8205, Apr. 2014, doi: 10.1364/OE.22.008205.

[3] R. Aguinaldo, Yiran Shen, and S. Mookherjea, "Large Dispersion of Silicon Directional Couplers Obtained via Wideband Microring Parametric Characterization," IEEE Photon. Technol. Lett., vol. 24, no. 14, pp. 1242-1244, Jul. 2012, doi: 10.1109/LPT.2012.2198639.

[4] J. R. Ong, R. Kumar, and S. Mookherjea, "Silicon microring-based wavelength converter with integrated pump and signal suppression," Opt. Lett., vol. 39, no. 15, p. 4439, Aug. 2014, doi: 10.1364/OL.39.004439.

[5] S. Mookherjea and A. Yariv, "Pulse propagation in a coupled resonator optical waveguide to all orders of dispersion," Phys. Rev. E, vol. 65, no. 5, p. 056601, Apr. 2002, doi: 10.1103/PhysRevE.65.056601.

[6] S. Mookherjea and A. Yariv, "Optical pulse propagation and holographic storage in a coupled-resonator optical waveguide," Phys. Rev. E, vol. 64, no. 6, p. 066602, Nov. 2001, doi: 10.1103/PhysRevE.64.066602.

[7] S. Mookherjea, D. S. Cohen, and A. Yariv, "Nonlinear dispersion in a coupled-resonator optical waveguide," Opt. Lett., vol. 27, no. 11, p. 933, Jun. 2002, doi: 10.1364/OL.27.000933.

[8] S. Mookherjea, "Using gain to tune the dispersion relation of coupledresonator optical waveguides," IEEE Photon. Technol. Lett., vol. 18, no. 5, pp. 715-717, Mar. 2006, doi: 10.1109/LPT.2006.871144.

[9] M. L. Cooper and S. Mookherjea, "Numerically-assisted coupled-mode theory for silicon waveguide couplers and arrayed waveguides," Opt. Express, vol. 17, no. 3, p. 1583, Feb. 2009, doi: 10.1364/OE.17.001583.

[10] S. Mookherjea, "Spectral characteristics of coupled resonators," $J$. Opt. Soc. Am. B, vol. 23, no. 6, p. 1137, Jun. 2006, doi: 10.1364/JOSAB.23.001137.

[11] J. R. Ong et al., "Low-power continuous-wave four-wave mixing in silicon coupled-resonator optical waveguides," Opt. Lett., vol. 36, no. 15, pp. 2964-2966, 2011.

[12] M. L. Cooper et al., "235-ring Coupled-Resonator Optical Waveguides," in Conference on Lasers and Electro-Optics 2010, San Jose, California, 2010, p. CTuHH3. doi: 10.1364/CLEO.2010.CTuHH3.

[13] M. L. Cooper, G. Gupta, J. S. Park, M. A. Schneider, I. B. Divliansky, and S. Mookherjea, "Quantitative infrared imaging of silicon-on-insulator 
microring resonators," Opt. Lett., vol. 35, no. 5, p. 784, Mar. 2010, doi: 10.1364/OL.35.000784.

[14] S. Mookherjea and M. A. Schneider, "Avoiding bandwidth collapse in long chains of coupled optical microresonators," Opt. Lett., vol. 36, no. 23, p. 4557, Dec. 2011, doi: 10.1364/OL.36.004557.

[15] M. L. Cooper and S. Mookherjea, "Modeling of Multiband Transmission in Long Silicon Coupled-Resonator Optical Waveguides," IEEE Photon. Technol. Lett., vol. 23, no. 13, pp. 872-874, Jul. 2011, doi: 10.1109/LPT.2011.2141657.

[16] J. R. Ong and S. Mookherjea, "Quantum light generation on a silicon chip using waveguides and resonators," Opt. Express, vol. 21, no. 4, p. 5171, Feb. 2013, doi: 10.1364/OE.21.005171.

[17] S. Mookherjea, "Semiconductor coupled-resonator optical waveguide laser," Appl. Phys. Lett., vol. 84, no. 17, pp. 3265-3267, Apr. 2004, doi: 10.1063/1.1719278.

[18] R. Kumar, M. Savanier, J. R. Ong, and S. Mookherjea, "Entanglement measurement of a coupled silicon microring photon pair source," Opt. Express, vol. 23, no. 15, p. 19318, Jul. 2015, doi: 10.1364/OE.23.019318.

[19] S. Mookherjea, J. R. Ong, X. Luo, and L. Guo-Qiang, "Electronic control of optical Anderson localization modes," Nature Nanotech, vol. 9, no. 5, pp. 365-371, May 2014, doi: 10.1038/nnano.2014.53.

[20] X. Wang, P. O. Weigel, J. Zhao, M. Ruesing, and S. Mookherjea, "Achieving beyond-100-GHz large-signal modulation bandwidth in hybrid silicon photonics Mach Zehnder modulators using thin film lithium niobate," APL Photonics, vol. 4, no. 9, p. 096101, Sep. 2019, doi: 10.1063/1.5115243.

[21] Y. Shen, I. B. Divliansky, D. N. Basov, and S. Mookherjea, "Perfect set-and-forget alignment of silicon photonic resonators and interferometers," in Optical Fiber Communication Conference/National Fiber Optic Engineers Conference 2011, Los Angeles, California, 2011, p. PDPC3. doi: 10.1364/OFC.2011.PDPC3.

[22] M. Savanier, R. Kumar, and S. Mookherjea, "Optimizing photon-pair generation electronically using a $p-i-n$ diode incorporated in a silicon microring resonator," Appl. Phys. Lett., vol. 107, no. 13, p. 131101, Sep. 2015, doi: 10.1063/1.4932047.

[23] S. Mookherjea and H. R. Grant, "High dynamic range microscope infrared imaging of silicon nanophotonic devices," Opt. Lett., vol. 37, no. 22, p. 4705, Nov. 2012, doi: 10.1364/OL.37.004705. 